\title{
The ability of orthodontists and oral/ maxillofacial surgeons to predict eruption of lower third molar
}

\author{
Aline do Carmo Bastos ${ }^{1}$, Joelma Bezerra de Oliveira', Karina Flexa Ribeiro Mello', Patrícia Botelho Leão', \\ Flavia Artese $^{2}$ and David Normando ${ }^{3^{*}}$
}

\begin{abstract}
Background: The aim of this study was to evaluate the ability of oral/maxillofacial surgeons (OMFSs) and orthodontists to predict third molar eruption by examining a simple panoramic radiograph in cases where full spontaneous eruption occurred.

Methods: Panoramic radiographs of 17 patients, 13-16 years of age, were obtained just after orthodontic treatment (T1), when the third molars were intraosseous. The radiographs at T1 were presented to 28 OMFSs and 28 orthodontists - who were asked to give a prognosis for the lower third molars on both sides $(n=34)$. The full spontaneous eruption of all third molars was clinically observed when patients were older than 18 years (T2). These teeth were clinically asymptomatic at $\mathrm{T} 1$ and $\mathrm{T} 2$.

Results: OMFSs decided by extractions in $49.6 \%$ of cases while orthodontists in $37.8 \%(p<0.001)$, when the radiographs were examined at T1. Agreement between OMFSs and orthodontists was excellent (Kappa $=0.76$, $p<0.0001$ ), as well as intragroup agreement for both OMFSs (Kappa $=0.83$ ) and orthodontists (Kappa $=0.96)$.

Conclusions: Despite a remarkable agreement for third molar prognosis, orthodontists and OMFSs were unable to predict lower third molar eruption by examining a simple panoramic radiograph. Both indicated extractions of a considerable number of spontaneously erupted asymptomatic teeth.
\end{abstract}

Keywords: Third molar, Impaction, Extraction, Dental eruption

\section{Background}

Third molar extraction is one of the most frequent procedures in oral surgery. Ten million teeth are extracted from approximately five million individuals every year in the USA [1]. The reason for third molar removal include the risk of impaction associated with caries, pericoronitis, periodontal defects in the distal surface of second molars, odontogenic cysts, and dental crowding [1-5]. A prospective study showed that general dentists recommended removal of third molars in $59 \%$ of participants, mainly to prevent future problems or because a third molar had an unfavorable orientation or was unlikely to erupt [6]. A recent systematic review evaluated the

\footnotetext{
* Correspondence: davidnormando@hotmail.com

${ }^{3}$ Department of Orthodontics, Federal University of Pará, Augusto Corrêa St., number 1, College of Dentistry, Belém, Pará 66.075-110, Brazil Full list of author information is available at the end of the article
}

prevalence of third molar impaction worldwide based on radiographic examination. Worldwide impaction prevalence was found to be $24.40 \%(95 \% \mathrm{CI}, 18.97$ to $30.80 \%$ ), which is much smaller than the percentage that undergoes clinical treatment for M3 problems [7].

The ideal moment to determine whether or not to remove third molars is also under debate, since impaction prediction has not been scientifically proven. Moreover, it is a daunting task to predict this biological condition with any degree of reliability [8]. Systematic reviews have reported that there is no evidence to support or refute prophylactic removal of asymptomatic impacted third molars, even in adults $[9,10]$. The scientific evidence contraindicates the prophylactic removal of third molars in order to prevent late lower anterior crowding $[9,10]$. However, in comparing the opinion of orthodontists and oral/maxillofacial surgeons (OMFSs), it became clear 
that both indicate prophylactic removal of third molars to prevent crowding $[11,12]$.

Although reports suggest that the predictive power of third molar eruption is low [3] and impacted third molars that remain static, with no changes in position or angulation over time are rare [13], professionals are still highly prone to indicate the extraction of these teeth, often in early adolescence. This study aimed to evaluate the ability of OMFSs, and orthodontists to predict third molar eruption by examining a simple panoramic radiograph in cases where the physiological eruption of third molars was known to have occurred.

\section{Methods}

This study was approved under \#498024 by the ethics committee for health science Institute of the Federal University of Pará. The sample included panoramic radiographs of 17 patients at the end of orthodontic treatment obtained from the databases of one private practice (D.N.). Inclusion criteria comprised individuals aged between 13 and 16 years, of both genders, treated without extractions and whose third molars erupted spontaneously years later (mean 6.4 years). All third molars were clinically asymptomatic at $\mathrm{T} 1$ and $\mathrm{T} 2$. Cases of agenesis, tooth loss, or tooth extractions for orthodontic purposes were excluded from the sample. Patients completed the orthodontic treatment between the years 2005 and 2010 (T1) and were reassessed between 2009 and 2014 (T2).

Fifty-six specialists-28 OMFSs and 28 orthodontists-were asked to provide, based on end-of-treatment panoramic radiographs (T1), their prognosis for the mandibular third molars present in those radiographs ( $n$ $=34$ ). These specialists were enrolled by voluntary response. The panoramic radiographs taken at the end of orthodontic treatment was randomly presented to each evaluator. Patient's age and sex were identified. The questionnaire included the following question: How would you approach the right and left lower third molars? Patient A's radiograph was duplicated in radiograph $E$ in order to evaluate method error, totaling 18 radiographic and 36 third molar evaluations.

The data collected were subjected to statistical analysis, at $p<0.05$, using BioEstat 5.3 software (Mamirauá Maintainable Development Institute, Belém, Pará, Brazil).

To evaluate intergroup and intragroup agreement, Kappa statistical analysis was used. Chi-square test was used to compare the distribution of responses between orthodontists and oral/maxillofacial surgeons.

\section{Results}

Diagnostic agreement between orthodontists and OMFSs was considered excellent for all patients, with a Kappa value of $0.76(p<0.0001$, Table 1$)$. Intragroup agreement
Table 1 Frequency of diagnostic response regarding extraction, monitoring or other alternatives for lower third molars given by orthodontists ( $n=28$ ) and oral/maxillofacial surgeons (OMFSs) $(n=28)$. Frequency distribution was evaluated by chi-square $\left(x^{2}\right)$ and agreement between evaluators by Kappa test

\begin{tabular}{lccc}
\hline & Orthodontists & OMFSs & Kappa $(p$ value $)$ \\
\hline Extraction & $360(37.8 \%)$ & $472(49.6 \%)$ & $0.76(<0.0001)$ \\
Monitoring & $586(61.6 \%)$ & $478(50.2 \%)$ & \\
Others & $6(0.6 \%)$ & $2(0.2 \%)$ & \\
Total & $952(100 \%)$ & $952(100 \%)$ & \\
Ortho $\times$ OMFSs $\left(x^{2}\right)$ & $25.56(p<0.0001)$, power $99 \%$ & \\
\hline
\end{tabular}

proved excellent for both oral/maxillofacial surgeons $($ Kappa $=0.83, p<0.0001)$ and orthodontists (Kappa $=$ $0.96, p<0.0001)$. Response unanimity was observed in two cases. One hundred percent of the OMFSs indicated extractions for both molars in patient $\mathrm{P}$, while all orthodontists indicated monitoring for patient $\mathrm{B}$.

Intergroup comparisons disclosed that the OMFSs tended to suggest extractions more often than orthodontists $(p<0.0001)$. Orthodontists indicated extraction in $37.8 \%$ of the cases, whereas OMFSs chose this procedure in almost half the cases (49.6 \%) (Table 1, Fig. 1a-c). It was observed that whenever an orthodontist indicated extraction of a third molar, the OMFSs had a $99.2 \%$ chance of agreement. And whenever an orthodontist decided to monitor a given case, the OMFS adopted the same approach in $81.1 \%$ of the cases.

The questionnaire included, in addition to the basic indications, i.e., extraction or monitoring, "another conduct" option to be described by the respondents. Eight responses selected this alternative $(0.8 \%)$. However, since the responses provided no justification, all eight answers were disregarded in the statistical analysis.

All lower third molars $(n=34)$ erupted spontaneously. Therefore, the most important finding of this research was that OMFSs and orthodontists are not able to make a reliable prognosis for lower third molar eruption using a panoramic radiograph, when these teeth have spontaneous eruption. Both groups of specialists tend to over- indicate extractions, when a third molar erupted spontaneously, mainly OMFSs (Table 1).

\section{Discussion}

When indicating extraction of third molars, dentists should have a justifiable reason, one that takes into account future treatment planning from an orthodontic, surgical, periodontal, and/or prosthetic point of view [11]. At the same time, a cost/benefit analysis should be carried out to justify the prophylactic removal of third molars, which should only be indicated with the purpose of preventing cases that involve pathological processes 

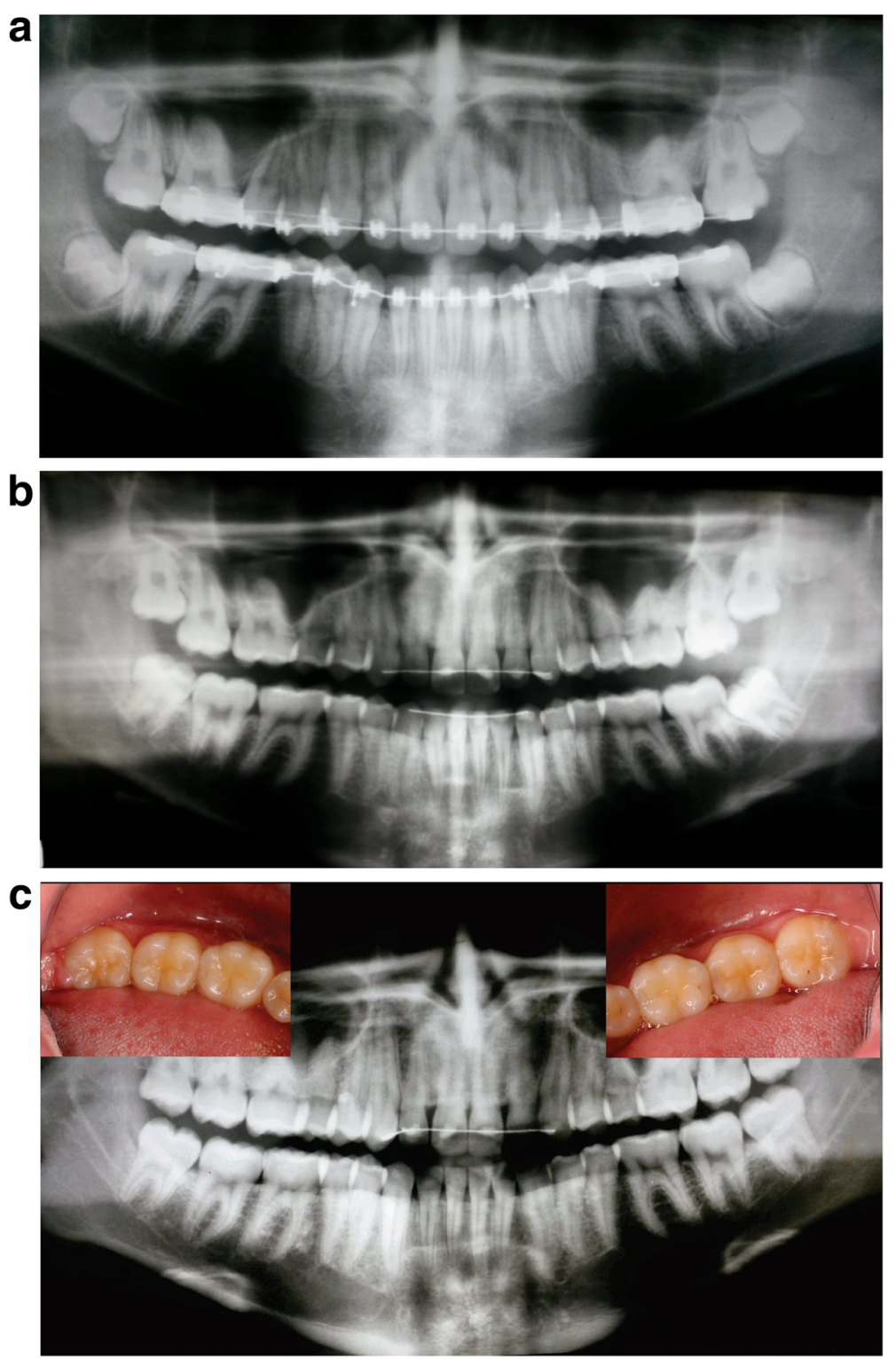

Fig. 1 Panoramic radiographs of patient G just before bracket removal (T1, 14 years - a), 4 years after orthodontic treatment (18 years-b) and after complete eruption of the lower third molars (T2, 22 years-c). For this patient, OMFSs indicated third molar extraction in $82 \%$ for the right side and $79 \%$ for the left side, while orthodontists suggested 65 and $62 \%$, respectively

such as root resorption or caries in the second molar, cysts, and pericoronaritis $[3,4,14,15]$.

The most relevant finding of this study was the inability of orthodontists and OMFSs to predict third molar prognosis when these teeth erupted spontaneously. Indication was given in $37.8 \%$ of cases by orthodontists and in $49.58 \%$ of cases by OMFSs. These findings corroborate indication by other authors $[16,17]$ who-in the absence of reliable predictors-suggested that ideally third molars should be monitored through periodic evaluations. The risk of developing diseases was the key motivator leading orthodontists and OMFs to indicate third molar extractions in our findings. This concept seems to be adopted in several countries $[6,16,17]$. General dentists in the USA recommend removal of third molars in $59 \%$ of cases [6]. A comparative analysis between the opinion of orthodontists and OMFSs, as regards to the role of third molars, found that $56.9 \%$ of OMFSs "often" or "sometimes" recommend prophylactic removal of third molars, while $64.4 \%$ of orthodontists "rarely" or "never" make this recommendation, underscoring a significant disagreement between these two 
specialists [3]. In this study, although both professionals showed significant agreement in their opinions (Kappa = 0.76), OMFSs tended to recommend more extractions than orthodontists $(p<0.0001)$. These findings support that orthodontists are more conservative than OMFSs and general dentists.

A survey comparing the views of clinicians and surgeons about the prophylactic removal of third molars conducted in Wales and Sweden found in the latter country a higher rate of third molar removal [5]. The authors explained the results by stating that in Wales, a protocol was developed which provides guidelines to decide whether or not to extract third molars, whereas such protocol is not widely accepted in Sweden. Arguably, the large number of third molar extractions currently performed is due to the lack of a protocol containing criteria that should be examined prior to recommending extraction. Despite the fact that the agreement for the responses given by the two groups of specialists was remarkable, the large number of extraction indications shows that the criteria used to support this decision must be reviewed.

An improvement in third molar position is observed in patients treated with premolar extractions [18-22]; however, when third molars are excessively tipped, they may remain impacted even if there is enough retromolar space [23]. If the probability of spontaneous eruption of third molars increases when premolars are extracted, it is likely that the reliability of prognosis is worse when these cases are assessed by orthodontists and surgeons. Thus, it seems necessary to evaluate the ability of the clinician to predict third molar eruption when orthodontic treatment involves premolar extractions.

Scientific evidence has shown that positional changes and eruption of lower third molar are unpredictable phenomena, whether in children and adolescents $[8,23]$ or even young adults [24]. Mandibular third molars at, or near to, the the occlusal plane and exhibiting vertical inclination were considered at highest risk for developing pericoronitis. Such third molars can be given high priority for prophylactic care due to the possibility of severe consequences of acute pericoronitis [25]. Furthermore, a higher incidence of dentigerous cysts may be associated with radiographically normal impacted lower third molar teeth [26]. Thus, prophylactic extractions of normal impacted lower third molars can be a treatment option even considering the risk of TMJ disorders [27]. The retrospective nature of this study increases the possibility of bias. A prospective follow-up study including different impaction severity and treatment outcomes should be considered, not only on an orthodontic perspective but also examining surgical complications after third molar removal [28].

\section{Conclusions}

- Despite a remarkable agreement for third molar prognosis, oral/maxillofacial surgeons and orthodontists were unable to predict third molars eruption by examining a single panoramic radiograph. Both indicated extractions for a considerable number of spontaneously erupted asymptomatic teeth, mainly oral surgeons.

- This paper encourages clinicians to re-evaluate their view on third molar extractions based on suggested radiograph guidelines. Other diagnostic methods that are also indicated for third molar eruption prediction should be investigated, such as longitudinal radiographs or 3D images.

Competing interests

The authors declare no financial support or conflicts of interest.

\section{Authors' contributions}

Conceived and designed the experiments: DN, KFRM, PBL. Collected data: $A C B, J B O$. Analyzed the data: ACB, JBO, KFRM, PBL, DN. Contributed material analysis tools: KFRM, PBL, FA, DN. Wrote the paper: ACB, JBO, KFRM, PBL, FA, DN. All authors read and approved the final manuscript.

\section{Author details}

'Department of Orthodontics, Brazilian Association of Dentistry, Trav. Marques de Herval, 2298, Belém, Pará 66.087-320, Brazil. ²Department of Orthodontics, Rio de Janeiro State University, Boulevard 28 de Setembro, 157, Rio de Janeiro, RJ CEP: 20551-030, Brazil. 'Department of Orthodontics, Federal University of Pará, Augusto Corrêa St., number 1, College of Dentistry, Belém, Pará 66.075-110, Brazil.

Received: 6 March 2016 Accepted: 22 June 2016

Published online: 11 July 2016

\section{References}

1. Friedman JW. The prophylactic extraction of third molars: a public health hazard. Am J Public Health. 2007:97:1554-9.

2. Katherine WL. Patient specific variables are a consideration in the decision to extract asymptomatic third molars. J Evid Base Dent Pract. 2012;12:92-4

3. Brokaw WC. The third molar question: when and why should we recommend removal? Virginia Dental J. 1991;68:18-21.

4. Lysell L, Rohlin M. A study of indications used for removal of the mandibular third molar. Int J Oral Maxillofac Surg. 1988;17:161-4.

5. Stanley HR, Alattar M, Collett WK, Stringfellow Jr HR, Spiegel EH. Pathological sequelae of "neglected" impacted third molars. J Oral Pathol. 1988;17:113-7.

6. Cunha-Cruz J, Rothen M, Spiekerman C, Drangsholt M, McClellan L, Huang GJ. Northwest practice-based research collaborative in evidence-based dentistry. Recommendations for third molar removal: a practice-based cohort study. Am J Public Health. 2014;104:735-43.

7. Carter K, Worthington S. Predictors of third molar impaction: a systematic review and meta-analysis. J Dent Res. 2016;95(3):267-76.

8. Richardson ER, Malhotra SK, Semenya K. Longitudinal study of three views of mandibular third molar eruption in males. Am J Orthod. 1984;86:119-29.

9. Costa MG, Pazzini CA, Pantuzo MC, Jorge ML, Marques LS. Is there justification for prophylactic extraction of third molars? A systematic review. Braz Oral Res. 2013;27:183-8.

10. Mettes TG, Ghaeminia H, Nienhuijs ME, Perry J, Van der Sanden WJ, Plasschaert AJ. Surgical removal versus retention for the management of asymptomatic impacted wisdom teeth. Cochrane Database Syst Rev. 2012:13:CD003879.

11. Lindauer SJ, Laskin DM, Tüfekçi E, Taylor RS, Cushing BJ, Best AM. Orthodontists' and surgeons' opinions on the role of third molars as a cause of dental crowding. Am J Orthod Dentofac Orthop. 2007;132:43-8. 
12. Gavazzi M, De Angelis D, Blasi S, Pesce P, Lanteri V. Third molars and dental crowding: different opinions of orthodontists and oral surgeons among Italian practitioners. Prog Orthod. 2014;15:60.

13. Phillips C, White RP. How predictable is the position of third molars over time? J Oral Maxillofac Surg. 2012;70:S11-4.

14. Kahl B, Gerlach KL, Hilgers RD. A long term follow-up, radiographic evaluation of asymptomatic impacted third molars in orthodontically treated patients. Int J Oral Maxillofac Surg. 1994;23:279-85.

15. Falci SG, de Castro CR, Santos RC, de Souza Lima LD, Ramos-Jorge ML, Botelho AM, Dos Santos CR. Association between the presence of partially erupted mandibular third molar and the existence of caries in the distal of the second molars. Int J Oral Maxillofac Surg. 2012;41:1270-4.

16. Knutsson K, Lysell L, Brickley M, Shepherd JP. Comparison of decisions regarding prophylactic removal of mandibular third molars in Sweden and Wales. Br Dent J. 2001:190:198-202.

17. Tüfekçi E, Svensk D, Kallunki J, Huggare J, Lindauer SJ, Laskin DM. Opinions of American and Swedish orthodontist about the role of erupting third molars as a cause of dental crowding. Angle Orthod. 2009;79:1139-42.

18. Miclotte A, Van Hevele J, Roels A, Elaut J, Willems G, Politis C, Jacobs R. Position of lower wisdom teeth and their relation to the alveolar nerve in orthodontic patients treated with and without extraction of premolars: a longitudinal study. Clin Oral Investig. 2014;18(7):1731-9.

19. Hauy RR, Cuoghi OA, Mendonça MR. Radiographic evaluation of the behavior of lower third molars in patients that were orthodontically treated with and without first premolars extractions. R Dental Press Ortodon Ortop Facial. 2007:12:73-84

20. Russell B, Skvara M, Draper E, Proffit WR, Philips C, White Jr RP. The association between orthodontic treatment with removal of premolars and the angulation of developing mandibular third molars over time. Angle Orthod. 2013;83:376-80.

21. Türköz C, Ulusoy C. Effect of premolar extraction on mandibular third molar impaction in young adults. Angle Orthod. 2013;83:572-7.

22. Artun J, Thalib L, Little RM. Third molar angulation during and after treatment of adolescent orthodontic patients. Eur J Orthod. 2005;27:590-6.

23. Richardson ME. The etiology and prediction of mandibular third molar impaction. Angle Orthod. 1977;47:165-72.

24. Hattab FN, Positional changes and eruption of impacted mandibular third molars in young adults. A radiographic 4-year follow-up study. Oral Surg Oral Med Oral Pathol Oral Radiol Endod. 1997:84:604-8.

25. Yamalik K, Bozkaya S. The predictivity of mandibular third molar position as a risk indicator for pericoronitis. Clin Oral Investig. 2008;12:9-14.

26. Wali GG, Sridhar V, Shyla HN. A study on dentigerous cystic changes with radiographically normal impacted mandibular third molar. J Maxillofac Oral Surg. 2012;11:458-65.

27. Camino Junior R, Manzi MR, Carvalho MF, Luz JG, Pimentel AC, Deboni MC Manual reduction of articular disc after traumatic extraction of mandibular third molar: a case report. Dental Press J Orthod. 2015;20(5):101-7.

28. Sverzut CE, Trivellato AE, Sverzut AT, Azenha MR, Yamaji MA, Pepato AO Retained third molars removal in a severely resorbed edentulous mandible. A case report. Braz Dent J. 2013:24:532-6.

\section{Submit your manuscript to a SpringerOpen ${ }^{\circ}$ journal and benefit from:}

- Convenient online submission

- Rigorous peer review

- Immediate publication on acceptance

- Open access: articles freely available online

- High visibility within the field

- Retaining the copyright to your article 